\title{
Hepatobiliyer Hastalıklarda Tanıya Yönelik Görüntüleme Yöntemlerinden Endoskopik Retrograd Kolanjiopankreatografi ve Manyetik Rezonans Kolanjiopankreatografinin Etkinliğinin Karşılaştırılması
}

\section{Comparison of the Effectiveness of Endoscopic Retrograde Cholangiopancreatography and Magnetic Resonance Cholangiopancreatography as Diagnostic Imaging Methods in Hepatobiliary Diseases}

\author{
Ahmed Ramiz Baykan ${ }^{*}$, Tahir Buran², Emre Gerçeker³, Hakan Yüceyar ${ }^{4}$, Serdar Tarhan5, Elmas Kasap², \\ Gökhan Pekindil ${ }^{5}$ \\ ${ }^{1}$ Sağlık Bilimleri Üniversitesi, Erzurum Bölge Sağlık Uygulama ve Araştırma Merkezi, \\ Gastroenteroloji Bölümü, İzmir, Türkiye \\ ${ }^{2}$ Manisa Celal Bayar Üniversitesi Gastroenteroloji Anabilim Dalı, Manisa, Türkiye \\ ${ }^{3}$ İzmir Özel Gazi Hastanesi, Gastroenteroloji Bölümü, İzmir, Türkiye \\ ${ }^{4}$ İzmir Özel Medicana Hastanesi, Gastroenteroloji Bölümü, İzmir, Türkiye \\ ${ }^{5}$ Manisa Celal Bayar Üniversitesi Radyoloji Anabilim Dalı, Manisa, Türkiye
}

e-mail: ahmedbaykan@gmail.com, tahir.buran@hotmail.com,dr.emre.gerceker@gmail.com, hakanyuceyar@gmail.com, sertarhan@yahoo.com, elmaskasap@yahoo.com,pekindilg@yahoo.com

ORCID: 0000-0001-6798-0240

ORCID: 0000-0001-7856-1287

ORCID: 0000-0001-7494-2912

ORCID: 0000-0003-1545-362X

ORCID: 0000-0002-7861-5115

ORCID: 0000-0002-4335-1156

ORCID: 0000-0001-5971-3994

*Sorumlu yazar/ Corresponding Author: Ahmed Ramiz Baykan

Gönderim Tarihi / Received: 26.11.2020

Kabul Tarihi / Accepted: 18.01.2021

DOI: $10.34087 /$ cbusbed. 826927

\section{Öz}

Giriş ve Amaç: Manyetik rezonans kolanjiopankreatografinin tanıya yönelik etkinliğini tanıda altın standart olarak kabul edilen endoskopik retrograd kolanjiopankreatografi ile kıyaslayarak değerlendirmeyi planladık.

Gereç ve Yöntemler: Celal Bayar Üniversite hastanesi gastroenteroloji kliniğine 2012-2014 yılları arasında başvurup, Manyetik rezonans kolanjiopankreatografi ve sonrasında endoskopik retrograd kolanjiopankreatografi yapilan hastaların dosyaları retrospektif olarak incelendi.

Bulgular: Çalışmaya 53 erkek, 57 kadın toplam 110 hasta dâhil edildi. Hastaların ortalama yaşı 63,56士16,897 olarak saptandı. Endoskopik retrograd kolanjiopankreatografi altın standart olarak alındığında ve kanülasyon sağlanamayan hastalar çıkarıldığında koledokolitizis için manyetik rezonans kolanjiopankreatografinin sensitivitesi $(\% 66,6)$, spesifitesi $(\% 82,8)$ olarak saptand. Manyetik rezonans kolanjiopankreatografinin pozitif prediktif değeri $(\% 86,9)$, negatif prediktif değeri $(\% 59,1)$ olarak saptandı. $5 \mathrm{~mm}$ ve büyük taşlar dikkate alındığında, manyetik rezonans kolanjiopankreatografinin duyarlılığının \%91,9’ a çıktığı görüldü. Hepatobiliyer malignitelerde, manyetik rezonans kolanjiopankreatografinin sensitivitesi $(\% 69,56)$, spesifitesi $(\% 100)$ olarak saptandi. Manyetik rezonans kolanjiopankreatografinin hepatobiliyer maligniteler için pozitif prediktif değeri $(\% 100)$, negatif prediktif değeri $(\% 91,13)$ olarak saptand. 
Sonuç: MRCP, hepatobiliyer sistem hastalıklarında tanısında yaygın kullanılan bir görüntüleme metodudur. Malign hastalıkların ve $6 \mathrm{~mm}$ den büyük safra yolu taşlarının tanısında oldukça etkili olmasına rağmen küçük safra yolu taşlarının saptanmasında tanı değeri düşmektedir..

Anahtar kelimeler: Egzersiz, Huzurevleri, Sedanter davranış, Yaşam kalitesi, Yaşlı.

\begin{abstract}
Objective: To evaluate the diagnostic effectiveness of magnetic resonance cholangiopancreatography by comparing it with endoscopic retrograde cholangiopancreatography, which is considered the gold standard in diagnosis.

Material and Methods: The results of patients who applied to the celal bayar university hospital gastroenterology clinic between 2012-2014 and who performed magnetic resonance cholangiopancreatography and endoscopic retrograde cholangiopancreatography were analyzed retrospectively.

Results: The study included a total of 110 patients ( 53 males and 57 females). The mean age of the patients was 63.56 \pm 16.897 years. When endoscopic retrograde cholangiopancreatography was taken as the golden standard and after patients with failed cannulation were excluded, the sensitivity and specificity of magnetic resonance cholangiopancreatography in the diagnosis of choledocholithiasis were determined as $66.6 \%$ and $82.8 \%$, respectively. The positive predictive value of magnetic resonance cholangiopancreatography was $86.9 \%$ and the negative predictive value was $59.1 \%$. For gallstones $\geq 5 \mathrm{~mm}$, the sensitivity of magnetic resonance cholangiopancreatography increased to $91.9 \%$. For hepatobiliary malignancies, the sensitivity and specificity of magnetic resonance cholangiopancreatography were determined to be $69.56 \%$ and $100 \%$, respectively, and the positive predictive value was $100 \%$ and negative predictive value was $91.13 \%$.

Conclusion: Magnetic resonance cholangiopancreatography is a widely used imaging method in the diagnosis of hepatobiliary system diseases. Although it is very effective in the diagnosis of malignant diseases and bile duct stones larger than $6 \mathrm{~mm}$, its diagnostic value decreases in the detection of small bile duct stones
\end{abstract}

Keywords: Endoscopic retrograde cholangiopancreatography, Gallstone, Magnetic resonance cholangiopancreatography.

1. Giriş
Endoskopik retrograd kolanjiopankreatografinin (ERCP) 1970'li yıllarda uygulanmaya başlaması ardından hepatobiliyer sistem hastalıklarının tanı ve tedavisinde yeni bir döneme girilmiş oldu [1]. İlerleyen dönemlerde hız kazanan teknoloji ile birlikte Alman araştırmacı Wallner ve arkadaşları tarafindan manyetik rezonans kolanjiyopankreatografi (MRCP) geliștirildi [2].

ERCP; duodenoskop ve röntgen ışınları yardımıyla kontrast madde verilerek pankreas, safra kesesi ve karaciğerin drenajını sağlayan kanalların görüntülenmesi yöntemidir [3]. ERCP görüntülemenin yanı sıra teröpatik girişime imkân vermesi nedeni ile altın standart olarak değerlendirilir [4]. Fakat her ne kadar günümüzde hastalar hastanelerde daha kısa süre izlenseler de [5], işlemin invaziv bir işlem olması ve hastanede işlem sonras1 takip gerektirmesi, komplikasyon ve hatta mortalite $(\% 0,1-1)$ riskinin olması bu işlemin dezavantajıdır [6]. Bu ve benzeri olumsuzluklar nedeniyle, hepatobiliyer sistem hastalarında MRCP, bir tanı yöntemi olarak giderek yayılmaktadır

MRCP, MR myelografi (MRM) ve MR ürografi (MRU) MR hidrografi (MRH) adı verilen bir grup benzer görüntüleme prensibine dayanan tetkikin bir parçasıdır[7]. MRCP' nin pankreatit ve kolanjitin akut atakları sırasında rahatlıkla kullanılabilmesi, darlığın hem proksimalindeki hem de distalindeki safra yollarını gösterebilmesi, konvansiyonel T1-T2 ağırlıklı görüntülerle birlikte ekstraduktal yapılarında gösterebilmesine olanak sunması ve biliyoenterik anastomozlarda darlığı tespit edebilmesi, invaziv olmaması ERCP ye kıyasla önemli avantajlarıdır [1].

MRCP nin tanı koymadaki yeterliliği hepatobiliyer hastalığın türüne (kolelitiyazis, malignite, benign darlık) bağlı olarak değişmektedir. MRCP ile ERCP arasında yapılan kıyaslamalı çalışmalarda MRCP' nin güvenilirliği konusunda çelişkili sonuçlar alınmaktadır $[8,9]$. Dolayısı ile MRCP ye duyulan güven azalmaktadır. Çalışmamızda hastanemizde hepatobiliyer sistem hastalıklardan dolayı ERCP yapılan hastalarda çekilmiş olan MRCP' nin tanı koymadaki yeterliliğini retrospektif olarak değerlendirdik.

\section{Materyal ve Metot}

Celal Bayar Üniversitesi Tıp Fakültesi Hafsa Sultan Hastanesi gastroenteroloji kliniğine 2012-2014 yılları arasında başvuran, laboratuvar ve klinik bulgular ile safra yolu hastalığı düşünülüp MRCP çekilen, sonrasında ileri tanı veya tedavi amaçlı ERCP ișlemi uygulanan hastaların dosyaları retrospektif olarak incelendi.

Çalışmaya 53 erkek 57 kadın toplam 110 kişi dahil edildi. Çalışma öncesi Celal Bayar Üniversitesi Tıp Fakültesi etik kurul onayı alınmıștır. (Tarih 29/01/2014 etik kurul no: 2047848634)

Klostrofobi, metalik protez veya başka nedenlerden dolayı MRCP çekilemeyen, ERCP yapılmış hastalar ve MRCP tetkik raporlarında tetkikin suboptimal olarak değerlendirildiği, görüntü kalitesinin kötü olduğu hastalar çalışmaya dahil edilmedi.

MRCP ișlemi Celal Bayar Üniversitesi hastanesi Radyoloji bölümünde, (1,5 T signa GE HDxt) marka cihaz ile gerçekleştirilmiştir. Lokalizasyon ve kalibrasyon görüntüleri sağlandıktan sonra axial 2D Fiasta breath-hold görüntüler alınmıştır. Sekans özellikleri; time echo: min Full,flip angle: 70, bandwith: 83.33, frequancy:256, phase:320, nex:1.00 tarama boyutu ise field of view:36, kesit kalınlığı: $5.0 \mathrm{~mm}$, spacing: 1.0 mm' dir. Oluşan görüntülere 3D MRCP RTr 
ASSET ve thick slab MRCP ASSET sekansları ile ince kesitlendirme yapılmış ve 3 boyutlu olarak rekonstrükte edilmiştir. Bu sekanslardan 3D MRCP RTr ASSET' in özellikleri; time echo: min, bandwith: 31.25, frequancy:320, phase:288, nex:1.00 tarama boyutu ise field of view:34, kesit kalınlığ1 $1.4 \mathrm{~mm}$, lacs per slab:66'dır. thick slab MRCP ASSET özellikleri ise; time echo: 1388.0, Tekrarlama zaman1: minimum, bandwith: 31.25, frequancy:448, phase:256, phase Field of view: 1.0, lacs before pause:1, freq DIR: unswap, shim auto, tarama boyutu ise field of view:32, kesit kalınlığ 40.0 mm, spacing:0.0' dir.

ERCP işlemi Celal Bayar Üniversitesi Tıp Fakültesi Hafsa Sultan Hastanesi gastroenteroloji bölümünde (Olympus TJF-240) marka cihaz ile yapılmıştır. ERCP tetkiki yan görüşlü endoskop kullanılarak gerçekleştirilmiştir. 6-8 saatlik açlık sonrasında floroskopi eşliğinde endoskopla duodenuma ulaşılmış ve papilla vateri' nin kanüle edilmesi sonrasında kontrast madde verilerek safra yolları görüntülenmiştir. Tetkik sırasında uygun görülen olgulara sfinkterotomi, balon ve/veya basket ile taş çıkarma, stent yerleştirme, biyopsi gibi işlemler uygulanmıştır. İşlemlerde (endamed) marka 3 lümenli çapı 7-5.5 F sfinkterotom, (medwork) marka litotripsi kateteri, (endamed) marka 3 lümen balon çap1 $16 \mathrm{~mm}$ olan $7 \mathrm{~F}$ çaplı katater sahip tas çıkarma balonu ve (endamed) marka çapı $2.3 \mathrm{~mm}$ basket çapı $30 \mathrm{~mm}$ olan taş çıkarma basketi kullanılmıştır.

MRCP ve ERCP tetkik raporları; safra kanallarında genişleme varlığı, safra kanallarında taş varlığı, safra kanallarında darlık varlığ darlığın iyi huylu veya kötü huylu olarak raporlanması temel alınarak karşılaştırılmıştır. Bu karşılaştırmada koledoğun en geniş olduğu yerde transvers çapının 7 mm'den fazla (hasta kolesistektomili ise $10 \mathrm{~mm}$ 'den fazla) olarak raporlanması genişleme olarak kabul edilmiştir. İntrahepatik safra kanallarının genişliği ise raporda belirtildiği taktirde dikkate alınmıştır.

ERCP raporlarının değerlendirmesinde taş sayısı veya seviyesi dikkate alınmamış sadece taşın varlığı veya yokluğu ve raporda belirtilen taşın boyutu dikkate alınmıştır. Birden fazla belirtilen taşlarda en büyük olanın raporlanan boyutu dikkate alınmıştır. Darlıklar genişlemiş kanallarda sonlanma veya çapta incelme olarak değerlendirilmiştir.

Elde edilen bulguların istatistiksel olarak karşılaştırılmasında IBM SPSS Statistics 20 paket programından yararlanılmıştır. ERCP ve MRCP raporları incelendikten sonra sensitivite, spesifite, pozitif prediktif değer ve negatif prediktif değerler hesaplanmıştır. Hesaplamalar yapılırken ERCP sonuçları altın standart olarak alınmıştır.

\section{Bulgular ve Tartışma \\ 3.1 Bulgular}

Çalışmaya 53 erkek $(\% 48,2), 57$ kadın $(\% 51,8)$ toplam 110 hasta dâhil edilmiştir. Hastaların yaş aralığı 20-91 arasında idi. Ortalama yaş $63,56 \pm 16,89$ olarak saptandı. Erkeklerde ortalama yaş $63,32 \pm 15,25$ kadınlarda $63,79 \pm 18,42$ idi.
ERCP yapılan hastalardan 9 erkek, 6 kadın toplam 15 hastada kanülasyon gerçekleştirilemedi. Kanülasyon gerçekleşemeyen bu hastaların MRCP görüntülerinde hastaların 4 tanesinde normal, 5 tanesinde koledokolitiyazis, 4 tanesinde şüpheli malignite, 1 tanesinde koledok dilatasyonu, 1 tanesinde pankeas başında kist, olarak rapor edildi. MRCP normal olarak yorumlanan 4 hastanın biliyer tipte ağrısının persiste olması üzerine ERCP işlemi planlanmıştı.

Kanülasyon sağlanamayan hastalar çıkarıldığında toplam 95 hastada yapılan ERCP' de en sik rastlanan safra yolu obstrüksiyonu nedeni koledokolitiyazisdir. Toplam 60 hastada koledokolitiyazis saptanmıştır. Diğer nedenler incelendiğinde 23 tanesinde malignite, 6 tanesi normal, 1 tanesinde kist hidatiğin safra yollarına açılması, 3 tanesinde biliyer kaçak, 2 hastada ise mirizzi sendromu olarak rapor edilmiştir (Tablo 1).

Tablo 1. Hastaların ERCP ve MRCP sonucu rapor edilen taniları

\begin{tabular}{|l|l|l|}
\hline & \multicolumn{1}{|c|}{$\begin{array}{c}\text { ERCP } \\
\text { sonuçları }\end{array}$} & \multicolumn{1}{|c|}{$\begin{array}{c}\text { MRCP } \\
\text { sonuçları }\end{array}$} \\
\hline Koledokolitiyazis & 60 & 51 \\
\hline Malignite & 23 & 20 \\
\hline Normal & 6 & 25 \\
\hline Biliyer Kaçak & 3 & 1 \\
\hline Mirizzi Sendromu & 2 & - \\
\hline $\begin{array}{l}\text { Kist Hidatik, } \\
\text { Biliyer Açılım }\end{array}$ & 1 & - \\
\hline $\begin{array}{l}\text { Kanülasyon } \\
\text { Olmayan }\end{array}$ & 15 & - \\
\hline $\begin{array}{l}\text { Koledok } \\
\text { Dilatasyonu }\end{array}$ & - & 10 \\
\hline $\begin{array}{l}\text { Koledoğa safra } \\
\text { kesesi dişında bası } \\
\text { tümör veya kist) }\end{array}$ & - & 3 \\
\hline Toplam hasta & 110 & 110 \\
\hline
\end{tabular}

MRCP raporları incelendiğinde en s1k raporlanan tanı olarak koledokolitiyazis görmekteyiz. Toplam 110 hastada, 51 tanesinde koledokolitiyazis, 25 tanesi normal, 20 tanesi malignite, 10 tanesinde safra yollarında dilatasyon, 3 tanesinde safra yollarına dıştan bası (2 tanesinde pankreas başında kist, 1 tanesinde apse), 1 tanesinde safra yollarında kaçak olarak raporlandığını görmekteyiz. Safra yollarında taş olarak raporlanan 51 hastanın 9 tanesi kolesistektomi öyküsü bulunmaktaydı, 34 tanesinde eş zamanlı olarak safra kesesinde de taş bulunmaktaydi.

ERCP' de koledokolitiyazis tespit edilen 60 hasta incelendiğinde, bu hastaların MRCP raporunda 17 $(\% 28,3)$ hastanın normal, $40 \quad(66,7)$ hastanın koledokolitiyazis, 3 (\%5) hastada ise koledokta dilatasyon dişında bir patoloji izlenmediği raporlanmıștır. Tam tersinden bakacak olursak MRCP' de safra yollarında taş olarak raporlanan 51 hastanın yapilan ERCP' sinde $40 \quad(\% 78,4)$ tanesinde koledokolitiyazis, $2(\% 3,9)$ tanesinde hepatobiliyer malignite, $2(\% 3,9)$ tanesi normal, $2(\% 3,9)$ tanesinde ise 
mirizzi sendromu düşünülmüştür (kalan 5 hastada ERCP de kanülasyon sağlanamadı). ERCP' de safra yolunda biliyer kaçak düşünülen 3 hastanın MRCP' sinde 2 tanesi normal, 1 tanesi tanısında biliyer kaçak olduğu görülmüştür.

ERCP' de hepatobiliyer malignite düşünülen 23 hastanın MRCP' sinde $16(\% 69,6)$ tanesinde hepatobiliyer malignite, $2(\% 8,7)$ hastada koledokolitiyazis, 5 hastada ise $(\% 21,7)$ diğer benign nedenler (dıştan bası, koledokta dilatasyon) saptanmıștır. MRCP' de hepatobiliyer malignite olarak yorumlanan toplam 20 hastanın ise 16, sinda ERCP de de hepatobiliyer malignite düşünülmüş, kalan 4 hastada ise kanülasyon gerçekleştirilememiş peruktan transhepatik kolanjiografi uygulanmıştır.

$\mathrm{Bu}$ sonuçlar 1şı̆̆ında, ERCP altın standart olarak alındığında ve kanülasyon sağlanamayan 15 hasta çıkarıldığında safra yollarında taş için MRCP' nin sensitivitesi $(\% 66,66)$, spesifitesi $(\% 82,85)$ olarak saptanmıştır. Yine MRCP' nin pozitif prediktif değeri $(\% 86,95)$, negatif prediktif değeri $(\% 59,18)$ olarak saptanmıştır (Tablo 2).

Tablo 2. MRCP' nin safra yolu taşı ve malignite tanısındaki sensitivite, spesifite, pozitif prediktif değer, negatif prediktif değerleri

\begin{tabular}{|l|l|l|l|l|}
\hline & SENSITIVITE & SPESIFITE & PPD & NPD \\
\hline Koledokolitiyazis & $\% 66,66$ & $\% 82,85$ & $\% 86,95$ & $\% 69,18$ \\
\hline Malignite & $\% 69,56$ & $\% 100$ & $\% 100$ & $\% 91,13$ \\
\hline
\end{tabular}

PPD:Pozitif prediktif değer NPD:Negatif Prediktif Değer

Hepatobiliyer malignitelerde, ERCP altın standart alındığında ve kanülasyon yapılamayan hastalar çıkarıldığında MRCP' nin sensitivitesi $(\% 69,56)$, spesifitesi (\%100) olarak saptanmıştır. MRCP' nin hepatobiliyer maligniteler için pozitif prediktif değeri (\%100), negatif prediktif değeri $(\% 91,13)$ olarak saptanmıştır.

İzole safra yolu dilatasyonu, obstrüksiyona yol açan tümör, darlık veya taş olmaksızın safra yollarında dilatasyon varlığını tanımlamaktadır. Çalışmamızda toplam 9 hastanın $\mathrm{MRCP}$ raporunda safra yolu dilatasyonu saptanmıştır. Bu hastaların ERCP raporları bakıldığında 3 hastada koledokolitiyazis, 4 hastada hepatobiliyer malignite, kalan 2 hastanın ise normal ERCP bulguları olarak yorumland.

\subsection{Tartışma}

ERCP hali hazırda safra yolu hastalıklarının görüntülenmesinde altın standart olarak değerlendirilmektedir [10]. MRCP' nin safra yollarının tanısal görüntülemesindeki önemi ise giderek artmaktadır. MRCP' nin hepatobiliyer patolojilerde yapılan çalışmalarda farklı sonuçlar elde edilmektedir $[9,11,12]$. Biz çalışmamızda Celal Bayar Üniversitesi Hafsa Sultan Hastanesi gastroenteroloji kliniğine başvurup MRCP ve ERCP uygulanan 110 hastay1 retrospektif olarak değerlendirdik. Sonuçta, MRCP' nin safra yolunda taş mevcudiyetini göstermede sensitivitesini $(\% 66,66)$, spesifitesi $(\% 82,85)$; Safra yolu malignitelerinde ise sensitivitesi $(\% 69,5)$, spesifitesi (\%100) olarak saptadik. Manyetik rezonans kolanjiyopankreatografi ile çeşitli tanısal hatalarla karşılaşılabilmektedir. normal fizyolojik değişikliklerin patoloji gibi görülmesi (örneğin, Oddi sfinkterinin kontraksiyonu tümör ya da taş tanısına neden olabilir), striktürlerin olduğundan daha abartılı görünmesi gibi çeşitli teknik artefaktlar ortaya çıkabilmektedir [13]. Ayrica artefakta neden olan sorunlardan en önemlilerden birisi hareket artefaktıdır (örneğin barsak hareketleri). Hareket artefaktlarının yanı sıra özellikle dilate kanallarda görülebilen akım artefaktı, anastamoz bölgesindeki klips artefaktı safra kanallarının sağlıklı bir şekilde değerlendirilmesini engelleyebilir. Kooperasyonu yetersiz hastalarda solunuma bağl1 artefaktlar, periton içi serbest sıvısı olan hastalarda ise zemin sinyal intensitesinin değişmesi ve görüntü kalitesinin bozulması bir sorun olarak zaman zaman karşımıza gelmektedir. Bütün bu olumsuzlukların bir sonucu olarak, MRCP de ufak taşların zaman zaman gözden kaçabileceği bilinmektedir [14]. Zidi ve arkadaşlarının 70 olguyu içeren çalışmasında koledokolitiyazis saptanmasında MRCP' nin duyarlılı̆̆ını \%57, spesifitesini \%100, Pozitif prediktif değeri \%100, negatif prediktif değeri \%50 olarak saptamıştır [15]. Çalışmasındaki duyarlılığın düşük olmasını olguların yaklaşık yarısında saptanan safra yolu taş boyutunun $6 \mathrm{~mm}$ 'den küçük olması olduğunu düşünüyoruz. Nitekim, safra yolarında $5 \mathrm{~mm}$ ve üzeri taş saptanan hastalar incelendiğinde, toplam 37 hastanın 34 $(\% 91,9)$ tanesinde ERCP' de safra yolundaki taş1 teyit etmiştir.

Safra yolu taş boyutu ile ilgili Zidi ve ark. tarafından yapılan çalışmada $6 \mathrm{~mm}$ ve daha küçük olan taşların MRCP de görülme duyarlılığ $1 \% 33$ olarak saptamışlardır [15]. Sonrasında Nandalur ve ark. benzer bir çalışma yapmış ve $6 \mathrm{~mm}$ ve daha küçük taşlarda MRCP' nin duyarlılığını \%64-68 arasında bulmuşlardır [16]. Bizim çalışmamızda bu oran \%35 olarak saptadık. (Toplam 28 hastanın 10 tanesinde MRCP taş izlendi) MRCP' nin ufak taşları göstermede başarısızlığı daha ince kesit 
görüntülerin alınması, hasta uyumunun sağlanması ile bir ölçüde azaltılabileceğini düşünüyoruz.

Büyük taşlar ile kolanjiyoselüler karsinomu ayırt etmek ERCP' de her zaman kolay olmamaktadır. Bu durum kontrast verilmediği için, T2 ağırlıklı görüntülerde taşların karakteristik hipointens görünümü nedeni ile MRCP' de de aynıdır [17]. Hepatobiliyer maligniteler görüntülemede genellikle dolum defekti, düzensiz darlıklar, double duct sign (pankreas kanseri için oldukça tanısal olan MRCP bulgusu), poststenotik genişlemeler şeklinde izlenmektedir. Yayınlanmış çalışmalarda hepatobiliyer malignitelerde MRCP' nin sensitivitesini \%60-94, spesifitesini ise \%85-100 arasında olduğunu bildirmektedir [18-23] . Çalışmamızda sensitiviteyi $(\% 69,5)$ spesifiteyi ise $(\% 100)$ olarak saptadık

MRCP' nin kontrastsız elde olunmasından dolayı darlığın benign veya malign ayrımı da her zaman yapılamamaktadır. Becker ve arkadaşları safra yollarında taş veya darlık şüphesi ile başvuran 108 olguyu retrospektif olarak değerlendirdikleri çalışmalarında safra yolu anormalliğini \%97-99 duyarlılık ve \%95-97 seçicilik ile saptamalarına rağmen, olguların üçte birinde benign- malign darlık ayrımının yapılamadığ bildirmişlerdir [24]. Bizim çalışmamızda da toplam 9 hastada bu ayrim yapılamadı, bu hastalar sonrasinda ERCP ile değerlendirildiğinde, 4 hastada hepatobiliyer malignite, kalan 5 hastada ise koledokolitiyazis ve diğer benign nedenler düşünüldü.

Erden ve arkadaşlarının yapmış olduğu çalışmada MRCP görüntülemede hidatik kistin deforme görünümü veya duvarında fokal defekt bulunmasını kist hidatiğin biliyer sisteme rüptürü açısından anlamlı bulmuşlar, MRCP' nin bu yönde sensitivitesini $(\% 91,7)$, spesifitesini $(\% 82,8)$ olarak hesaplamışlardır [25]. Çalışmamızda MRCP ile sadece 1 hastada kist hidatiğin biliyer açılımını saptandı. $\mathrm{Bu}$ hastanın ERCP sonucu MRCP ile korale olduğu görüldü.

Mirizzi Sendromu tanı yollarından biri MRCP' dir. MRCP ile safra taşı lokalizasyonu, inflamatuar değişiklikler değerlendirilebilir. Fakat sistik kanal ve ana hepatik kanalın anatomik ilişkisinden dolayı taş her zaman görülemeyebilir. ERCP yine tanıda altın standart olarak kabul edilmektedir. MRCP' nin mirizzi sendromu tanısında sensitivitesi \%96, spesifitesi \%93,5 civarındadır [26]. Bizim çalışmamızda ERCP ile mirizzi düşünülen 2 hastada MRCP ile taşın uygun lokalizasyonu yapılamamış, koledokolitiyazis olarak yorumlanmıştır. MRCP safra yolu operasyonlarından sonra safra kaçağını görüntülemek için de sıklıkla kullanılmaktadır. Bu yönde özellikle kontrast maddelerle (gadobenate dimeglumine, gadolinium ethoxybenzyl diethylenetriamine pentaacetic acid gibi) uygulandığında tanı değeri yükselmektedir (sensitivite $\% 59, \quad$ kontrastl görüntülemede sensitivite \%79) [27]. Cieszanowski A ve ark. 2013 yılında yayınlanan bir çalışmasında safra yolu kaçağ 1 olan 34 hastada kontrast madde olan gadoxetic acid disodium (Gd-EOB-DTPA) kullanarak safra yolunda kaçağını görüntülemeyi hedeflemişlerdir. Çalışmalarında MRCP' nin bu yönde sensitivitesini \%96,4 spesifitesini \%100 olarak saptamışlardır [28].
Çalışmamızda ERCP ile saptanan toplam 3 biliyer kaçak hastasi oldu. $\mathrm{Bu}$ hastaların kontrast verilmeden yapilan MRCP sonuçlarında 2 hastada normal, 1 hastada ERCP ile korale olarak raporland1.

Hasta sayımızın safra yolu taşı dışındaki patolojilerde az olması çalışmamızın en büyük kısıtlaması idi. Ek olarak çalışmanın retrospektif tarzda olması sonucu, gerekli verilere ulaşamama, MRCP raporlarının tek bir kişi tarafından değerlendirilmemesi çalışmanın diğer kısıtlamalarını oluşturmaktaydı.

\section{Sonuç}

MRCP, hepatobiliyer sistem hastalıklarında tanısında yaygın kullanılan bir görüntüleme metodudur. Malign hastalıkların ve $6 \mathrm{~mm}$ den büyük safra yolu taşlarının tanısında oldukça etkili olmasına rağmen küçük safra yolu taşlarının saptanmasında tanı değeri düşmektedir.

\section{Referanslar}

1. Albert, J.G, Riemann, J.F. ERCP and MRCP - When and why, Best Practice and Research, Clinical Gastroenterology, 2002, 16(3), 399419.

2.Wallner, B.K, Schumacher, K.A, Weidenmaier, W, Friedrich, J.M Dilated biliary tract: Evaluation with MR cholangiography with a T2 weighted contrast-enhanced fast sequence, Radiology, 1991, 181(3), 805-8.

3.Ceylan, G, Yavaşcaoğlu, B, Korfali, G, Fatma, N, Kaya, B, Moğol, $\mathrm{G}$, et al, Endoskopik Retrograd Kolanjiopankreatografi İșlemi İçin Bilinçli Sedasyon Uygulamasında Propofol ile Deksmedetomidinin Hemodinami ve Kognitif Fonksiyonlara Etkisinin Karşılaştırılması, Uludağ Üniversitesi Tip Fakültesi Dergisi, 2010, 10.

4.Kaltenthaler, E.C, Walters, S.J, Chilcott, J.B, Blakeborough, A, Vergel, Y.B, Thomas, S.M, MRCP compared to diagnostic ERCP for diagnosis when biliary obstruction is suspected: A systematic review. BMC Medical Imaging. 2006, Vol. 6.

5.Dursun, H.B, Yılmaz, E, Batın Cerrahisi Yapılan Hastaların Öğrenim Gereksinimleri, Celal Bayar üniversitesi Sağllk Bilimleri Enstitüsü Dergisi, 2015, 2.

6.Varghese, J.C, Farrell, M.A, Courtney, G, Osborne, H, Murray, F.E, Lee, M.J, A prospective comparison of magnetic resonance cholangiopancreatography with endoscopic retrograde cholangiopancreatography in the evaluation of patients with suspected biliary tract disease, Clinical Radiology, 1999, 54(8), 513 20 .

7.Jara, H, Barish, M.A, Yucel, E.K, Melhem, E.R, Hussain, S, Ferrucci, J.T, MR hydrography: Theory and practice of static fluid imaging, American Journal of Roentgenology, 1998,170(4), 873-82.

8.Vaníček, J, Kyselová, H, Kianička, B, Mikulicová, R, Bajgarová, B Trna, J, et al, MRCP a ERCP v diagnostice choledocholitiázy [Comparison of MRCP a ERCP in diagnosis of choledocholithiasis], Vnitrni Lekarstvi, 2013, 59(5), 357-60.

9.Rahman, R, Ju, J, Shamma's, J, Goebel, S, Sundaram, U, Correlation between MRCP and ERCP findings at a tertiary care hospital, The West Virginia medical journal, 2010, 106(5), 14-9.

10.Soto, J.A, Barish, M.A, Yucel, E.K, Siegenberg, D, Ferrucci, J.T, Chuttani, R, Magnetic resonance cholangiography: Comparison with endoscopic retrograde cholangiopancreatography, Gastroenterology, 1996, 110(2), 589-97.

11. Mandelia, A, Gupta, A.K, Verma, D.K, Sharma, S, The Value of Magnetic Resonance Cholangio-Pancreatography (MRCP) in the Detection of Choledocholithiasis, Journal of clinical and diagnostic research, 2013, 7(9).

12. Holzknecht, N, Gauger, J, Sackmann, M, Thoeni, R.F, Schurig, J, Holl, J, et al, Breath-hold MR cholangiography with snapshot techniques: Prospective comparison with endoscopic retrograde cholangiography, Radiology, 1998, 206(3), 657-64.

13. Düşünceli, E, Erden, A, Erden, I, Anatomic variations of the bile ducts: MRCP findings, Tanisal ve girisimsel radyoloji Tibbi Goruntuleme ve Girisimsel Radyoloji Dern yayin organi, 2004 ,10(4), 296-303. 
14. Srinivasa, S, Sammour, T, McEntee, B, Davis, N, Hill, A.G, Selective use of magnetic resonance cholangiopancreatography in clinical practice may miss choledocholithiasis in gallstone pancreatitis. Canadian Journal of Surgery, 2010, 53(6):403-7.

15. Zidi, S.H, Prat, F, Le Guen, O, Rondeau, Y, Rocher, L, Fritsch, J, et al, Use of magnetic resonance cholangiography in the diagnosis of choledocholithiasis: prospective comparison with a reference imaging method, Gut, 1999, 44(1), 118-22.

16. Nandalur, K.R, Hussain, H.K, Weadock, W.J, Wamsteker, E.J, Johnson, T.D, Khan, A.S, et al, Possible biliary disease: diagnostic performance of high-spatial-resolution isotropic 3D T2-weighted MRCP, Radiology, 2008, 249(3), 883-90.

17. Kats, J, Kraai, M, Dijkstra, A.J, Koster, K, Ter Borg, F, Hazenberg, H.J.A, et al, Magnetic resonance cholangiopancreaticography as a diagnostic tool for common bile duct stones: a comparison with ERCP and clinical follow-up, Digestive Surgery, 2003, 20(1), 32-7.

18. Catalano, C, Pavone, P, Laghi, A, Panebianco, V, Scipioni, A, Fanelli, F, et al, Pancreatic adenocarcinoma: combination of MR imaging, MR angiography and MR cholangiopancreatography for the diagnosis and assessment of resectability, European journal of Radiology, 1998, 8(3), 428-34.

19. Diehl, S.J, Lehmann, K.J, Gaa, J, Meier-Willersen, H.J, Wendl, K, Georgi, M, The value of magnetic resonance tomography (MRT), magnetic resonance cholangiopancreatography (MRCP) and endoscopic retrograde cholangiopancreatography (ERCP) in the diagnosis of pancreatic tumors, Rofo: Fortschritte auf dem Gebiete der Rontgenstrahlen und der Nuklearmedizin, 1999, 170(5), 463-9.

20. Kim, M.J, Mitchell, D.G, Ito, K, Outwater, E.K, Biliary dilatation: differentiation of benign from malignant causes--value of adding conventional MR imaging to MR cholangiopancreatography, Radiology, 2000, 214(1), 173-81.

21. Vogl, T.J, Schwarz, W.O, Heller, M, Herzog, C, Zangos, S, Hintze, $\mathrm{RE}$, et al, Staging of Klatskin tumours (hilar cholangiocarcinomas) comparison of MR cholangiography, MR imaging, and endoscopic retrograde cholangiography, European journal of Radiology, 2006 , 16(10), 2317-25.

22. Adamek, H.E, Albert, J, Weitz, M, Breer, H, Schilling, D, Riemann, J.F, A prospective evaluation of magnetic resonance cholangiopancreatography in patients with suspected bile duct obstruction, Gut, 1998, 43(5), 680-3.

23. Lopez Hänninen, E, Amthauer, H, Hosten, N, Ricke, J, Böhmig, M, Langrehr, J, et al, Prospective evaluation of pancreatic tumors: accuracy of MR imaging with MR cholangiopancreatography and MR angiography, Radiology, 2002, 224(1), 34-41.

24. Becker, C.D, Grossholz, M, Becker, M, Mentha, G, de Peyer, R, Terrier, F, Choledocholithiasis and bile duct stenosis: diagnostic accuracy of MR cholangiopancreatography, Radiology, 1997 , 205(2), 523-30.

25. Erden, A, Ormeci, N, Fitoz, S, Erden, I, Tanju, S, Genç Y, Intrabiliary rupture of hepatic hydatid cysts: diagnostic accuracy of MR cholangiopancreatography, AJR American Journal of Roentgenology, 2007, 189(2).

26. Yun, E.J, Choi, C.S, Yoon, D.Y, Seo, Y.L, Chang, S.K, Kim, J.S, et al, Combination of magnetic resonance cholangiopancreatography and computed tomography for preoperative diagnosis of the mirizzi syndrome, Journal of Computer Assisted Tomography, 2009, 33(4), 636-40.

27. Kantarci, M, Pirimoglu, B, Karabulut, N, Bayraktutan, U, Ogul, H Ozturk, G, et al, Non-invasive detection of biliary leaks using GdEOB-DTPA-enhanced MR cholangiography: Comparison with T2weighted MR cholangiography, European journal of Radiology, $2013,23(10), 2713-22$.

28.Cieszanowski, A, Stadnik, A, Lezak, A, Maj, E, Zieniewicz, K, Rowinska-Berman, K, et al, Detection of active bile leak with Gd EOB-DTPA enhanced MRcholangiography: Comparison of 20?25 min delayed and 60?180 mindelayed images, Europan Journal of Radiology, 2013, 82(12), 2176-82. http://edergi.cbu.edu.tr/ojs/index.php/cbusbed isimli yazarın CBU-SBED başlıklı eseri bu Creative Commons Alıntı-Gayriticari4.0 Uluslararası Lisansı ile

\section{0}

